\title{
Evidence for a Functional Link between Profilin and CAP in the Yeast S. cerevisiae
}

\author{
Anne Vojtek," Brian Haarer, $\uparrow$ Jeffrey Field," \\ Jeffrey Gerst," Thomas D. Pollard, $\ddagger$ \\ Susan Brown, + and Michael Wigler* \\ *Cold Spring Harbor Laboratory \\ P.O. Box 100 \\ Cold Spring Harbor, New York 11724-2208 \\ tDepartment of Anatomy and Cell Biology \\ The University of Michigan Medical School \\ Ann Arbor, Michigan 48109 \\ łDepartment of Cell Biology and Anatomy \\ The Johns Hopkins University School of Medicine \\ Baltimore, Maryland 21205
}

\section{Summary}

CAP is a component of the S. cerevisiae adenylyl cyclase complex. The $\mathrm{N}$-terminal domain is required for cellular RAS responsiveness. Loss of the C-terminal domain is associated with morphological and nutritional defects. Here we report that cap cells bud randomly and are defective in actin distribution. The morphological and nutritional defects associated with loss of the CAP C-terminal domain are suppressed by overexpression of PFY, the gene encoding profilin, an actin- and polyphosphoinositide-binding protein. The phenotype of cells lacking PFY resembles that of cells lacking the CAP C-terminal domain. Study of mutated yeast profilins and profilins from Acanthamoeba suggests that the ability of profilin to suppress cap- cells is dependent upon a property other than, or in addition to, its ability to bind actin. This property may be its ability to bind polyphosphoinositides. We propose that CAP and profilin provide a link between growth signals and remodeling of the cellular cytoskeleton.

\section{Introduction}

RAS proteins form a class of low molecular weight guanine nucleotide-binding proteins that have been highly conserved in evolution and are thought to participate in signal transduction pathways that regulate growth and differentiation (Barbacid, 1987). Despite extensive studies, the identity of a RAS effector has been delineated in only one organism to date, the yeast Saccharomyces cerevisiae (Toda et al., 1985). In this yeast, RAS activates adenylyl cyclase activity in a complex that consists of at least two proteins: the adenylyl cyclase catalytic subunit, encoded by the CYR1 gene; and an adenylyl cyclase-associated protein, encoded by the CAP gene (Field et al., 1990; Fedor-Chaiken et al., 1990)

Previous results indicate that CAP is a bifunctional signal transduction protein. The $\mathrm{N}$-terminal domain of CAP, amino acids 1 to 168 , appears to be both necessary and sufficient for full cellular responsiveness to mutant activated RAS proteins (Gerst et al., 1991). Disruption of the $C$-terminal domain of CAP in a wild-type RAS background is associated with nutritional and morphological defects: an inability to grow on rich medium (reflecting sensitivity to an excess of certain amino acids, particularly valine); sensitivity to nitrogen starvation; temperature sensitivity on minimal medium; and abnormally enlarged and round cells (Field et al., 1990; Gerst et al., 1991). These defects are complemented by overexpression of the C-terminal domain of CAP, amino acids 368 to 526 (Gerst et al., 1991), but are not suppressed by overexpression of positive or negative regulatory components of the RAS/adenylyl cyclase pathway, including CAMP phosphodiesterases, adenylyl cyclase itself, the regulatory or catalytic subunits of the cAMP-dependent kinase (Field et al., 1990), or the $\mathrm{N}$-terminus of CAP (Gerst et al., 1991).

We have selected yeast genes on multicopy plasmids capable of suppressing the phenotypes that result from loss of the C-terminal functions of CAP. One of the genes we have found in our search is $P F Y$, which encodes profilin (Magdolen et al., 1988).

Profilins have been characterized from a variety of sources, including human platelets, the protozoan Acanthamoeba, and the yeast $S$. cerevisiae. In all cases examined, profilins bind to, decrease ATP hydrolysis by, and inhibit the polymerization of actin monomers in vitro (Pollard and Cooper, 1986; Stossel et al., 1985; Haarer et al., 1990). Additionally, profilins from platelets and the protozoan Acanthamoeba bind polyphosphoinositides in vitro (Lassing and Lindberg, 1985, 1988; Machesky et al., 1990). When profilin is bound to polyphosphoinositides, it cannot bind actin monomers nor block actin polymerization (Lassing and Lindberg, 1985, 1988). These in vitro biochemical properties have led to the speculation that profilin is a component of a signal transduction cascade that modulates the cytoskeleton (Lassing and Lindberg, 1985,1988 ). Other molecules within the cell, such as gelsolin, also bind actin and polyphosphoinositides (Janmey and Stossel, 1987) and are candidates for such regulatory molecules.

Disruption of the S. cerevisiae PFY gene provided the first genetic evidence that profilin function is required for cytoskeletal integrity. Cells lacking profilin are abnormally enlarged and round, do not contain actin cables, and bud randomly from the cell surface (Haarer et al., 1990). Similar defects in cell morphology and in actin distribution are seen in cells that overexpress the actin-binding protein ABP1 (Drubin et al., 1988) as well as in cells with aberrations in other known or suspected actin-binding proteins (Adams et al., 1989; Amatruda et al., 1990).

Profilins have also been reported to protect polyphosphoinositides from hydrolysis by phospholipase $C-\gamma 1$ in vitro (Goldschmidt-Clermont et al., 1990; Machesky et al., 1990). This property correlates with the ability of profilins to bind polyphosphoinositides (Goldschmidt-Clermont et al., 1990; Machesky et al., 1990). In Acanthamoeba there are two profilins, isoform I and isoform II. Both bind actin with equal affinities, but isoform II binds polyphosphoinositides with 10- to 50-fold higher affinity than does isolorm I, 


\begin{tabular}{lll}
\hline Table 1. Strain Descriptions & \\
\hline Strain & Genotype & Reference \\
\hline SP1 & MATa leu2 ura3 trp1 his3 ade8 can1 & CSHL collection \\
SKN32 & MATa leu2 ura3 trp1 ade8 can1 cap::HIS3 & Field et al., 1990 \\
SKN37 & MATa leu2 ura3 trp1 ade8 can1 RAS2val19 cap::HIS3 & Field et al., 1990 \\
PL1 & ura3 trp1 his ade8 can1 pfy::LEU2 & This study \\
PU1 ${ }^{b}$ & leu2 trp1 his ade8 can1 pfy::URA3 & This study \\
\hline
\end{tabular}

a Segregant from the diploid SP1/DC124 (CSHL collection) that had been transformed with the BamHI-HIndlll fragment of pHBL1-1 to disrupt PFY. - Segregant from the diploid SP1/DC124 that had been transformed with the BamHI-Hindll fragment of pU2-9 to disrupt PFY.

and commensurably protects polyphosphoinositides from hydrolysis by phospholipase $\mathrm{C}$. These in vitro biochemical properties have led to the speculation that some profilins might participate in the generation of second messengers derived from the phosphatidylinositol cycle.

Further support for a role of profilin in phosphoinositide metabolism comes from more recent in vitro studies (Goldschmidt-Clermont et al., 1991). Growth factors such as platelet-derived growth factor and epidermal growth factor activate receptors that are tyrosine-specific protein kinases, and induce elevations of inositol phosphates (Berridge et al., 1984; Wahl and Carpenter, 1988; Wahl et al., 1989). Phospholipase $\mathrm{C}-\gamma 1$ also becomes tyrosine phosphorylated (Wahl et al., 1988, 1989; Meisenhelder et al., 1989; Margolis et al., 1989). This phosphorylation does not increase the activity of phospholipase $\mathrm{C}-\gamma 1$ toward polyphosphoinositides in artificial phospholipid bilayers, but it does render the polyphosphoinositides bound to profilin accessible to digestion (Goldschmidt-Clermont et al., 1991). One model suggested by this work is that the subcompartment of phosphoinositides bound to molecules like profilin is a source for second messengers, and is especially sensitive to growth signals that induce tyrosine phosphorylation of phospholipase $\mathrm{C}-\gamma 1$.

The yeast profilin gene on a multicopy plasmid suppresses both the nutritional and morphological defects of CAP-deficient cells. Cells lacking PFY have a phenotype that resembles in kind, though not in intensity, the phenotype of cells lacking CAP. Hence, we propose that CAP and profilin are components of the same signaling pathway. Our studies of mutant yeast profilins and the two profilin isoforms of Acanthamoeba have partially separated the functions of profilin: the ability to suppress the defects of PFY-deficient strains and the ability to suppress the loss of CAP function. Acanthamoeba profilin isoform II, the one with high affinity for polyphosphoinositides, can suppress more efficiently the defects of CAP-deficient strains than does isoform I, and hence we propose that CAP, in addition to its role in mediating the effects of activated RAS, may be a component of a polyphosphoinositide signal transduction pathway in $\mathrm{S}$. cerevisiae.

\section{Results}

Isolation of the S. cerevisiae Profilin Gene To isolate genes that when overexpressed suppress the sensitivity of cap- strains to growth on rich medium, we used the strain SKN37 (cap::HIS3; see Table 1), in which virtually the entire coding capacity of CAP has been deleted. SKN37 was transformed with a library of yeast genomic DNA in a $2 \mu$ high copy expression vector. Leu ${ }^{+}$transformants were scored for their ability to grow on rich medium (YPD) by replica plating. Out of approximately $4000 \mathrm{Leu}^{+}$transformants analyzed, 12 exhibited a plasmid-dependent ability to grow on YPD. Plasmids were rescued from these transformants, and by Southern hybridization analysis, ten of those cross-hybridized. One of the ten was selected for further analysis. A restriction map of the smallest complementing region of this plasmid is shown in Figure 1A. The $1.8 \mathrm{~kb}$ region between the BamHI and HindlII sites was sequenced and found to contain the entire yeast PFY gene, encoding profllin (Magdolen et al., 1988). The plasmid was named pPFY. Deletion of the profilin open reading frame resulted in a plasmid unable to suppress the sensitivity of SKN37 to growth on rich medium (data not shown).

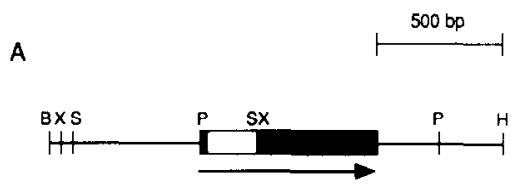

B

1 MSWOTYVDTNLVGTGAVTOAAILGLDG NTWATSAGFAVTE AOGOTLASAFNNADP IRASGEDL $111+11111111111111111: 111$ 1 $111111111: 11: 1 \quad 1111 \quad 1 \quad 111: 11$ 2 MSWOTYVDTNLVGTGAVTOAAIIGHDG NTWATSAGEAVSPANGAaLANAFKDATA IRSMGEI 3 MSWQAYTD NLIGTGKVDKAVIYSRAGDAVWATSGGLS LQRNE IGEIVQGFDNPAGLQSNGLH I

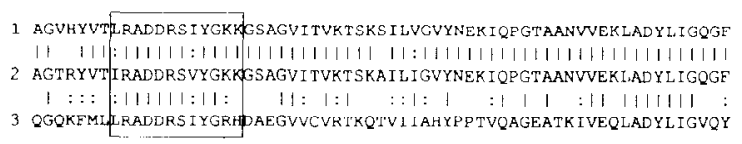

Figure 1. Restriction Map of the PFY Gene and a Comparison of Profilin Proteins

(A) Restriction map of the smallest complementing fragment of pPFY. Solid bars denote the location of the profilin open reading frame; open bar denotes the $208 \mathrm{bp}$ intron. The arrow indicates the direction of transcription. Restriction enzymes are abbreviated as follows: $B$ BamHI; H, HindlII; S, Sall; P, Pvul; $X, X$ hol.

(B) A comparison of profilin proteins. The profilin proteins were aligned with the IntelliGenetics, Inc. GENALIGN program. 1, Acanthamoeba profilin isoform I (Pollard and Rimm, 1991). 2, Acanthamoeba profilin isoform II (Pollard and Rimm, 1991). 3, S. cerevisiae profilin. Vertical bars indicate identity; colons indicate simllarlty, using the JimenezMontano and Zamora-Cortina alphabet. Similar amino acids are grouped as follows: V, L, I, M; F, Y, W; K, R; E, D; Q, N; S, T; and A, $G$. Box indicates region analyzed by site-directed mutagenesis (see Table 3). 

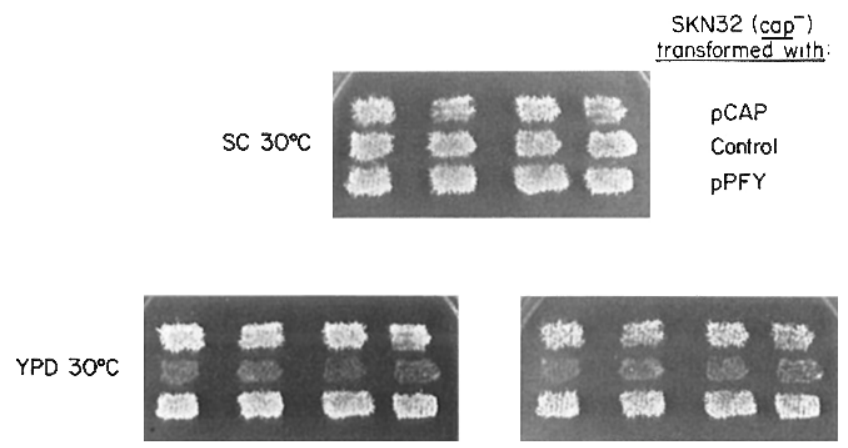

$\operatorname{sc~} 37^{\circ} \mathrm{C}$
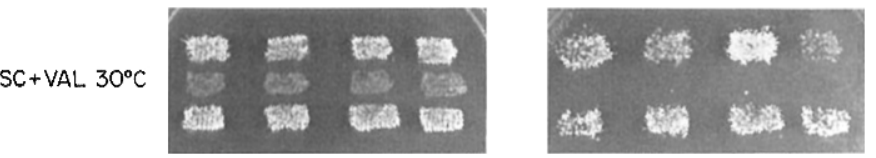

Figure 2. Suppression of cap- Phenotypes by PFY

SKN32 was transformed with the indicated plasmids and plated onto $\mathrm{SC}$ - Leu plates. Independent transformants were patched onto $\mathrm{SC}-$ Leu plates, incubated $3-4$ days at $30^{\circ} \mathrm{C}$ and then replica plated to the following plates, which were incubated at the specified temperatures: $\mathrm{SC}$ - Leu at $30^{\circ} \mathrm{C}\left(\mathrm{SC} 30^{\circ} \mathrm{C}\right)$; YPD at $30^{\circ} \mathrm{C}$; $\mathrm{SC}-$ Leu with $6.5 \mathrm{mM}$ valine at $30^{\circ} \mathrm{C}$ $\left(\mathrm{SC}+\mathrm{VAL} 30^{\circ} \mathrm{C}\right) ; \mathrm{SC}-$ Leu at $37^{\circ} \mathrm{C}\left(\mathrm{SC} 37^{\circ} \mathrm{C}\right)$; and $\mathrm{SC}-\mathrm{Leu}$ after a 7 day starvation on synthetic medium lacking a nitrogen source $(-\mathrm{Ni}$ trogen).
We next examined the ability of high copy plasmids expressing profilin to suppress the other defects in CAPdeficient strains. In the experiment shown in Figure 2, we transformed the strain SKN32 (cap::HIS3) with pPFY. pPFY suppressed these phenotypes of SKN32: inability to grow on YPD, sensitivity to valine, temperature sensitivity on synthetic medium, and sensitivity to nitrogen starvation. Thus overexpression of profilin suppresses the growth and nutritional defects associated with disruptions of the C-terminus of CAP. To explore the ability of profilin to complement the functions of the $\mathrm{N}$-terminus of CAP, we transformed SKN37 with pPFY and subjected transformants to a heat shock. SKN37 contains the RAS2 ${ }^{\text {val19 }}$ allele, which renders $C A P^{m}$ strains heat shock sensitive. SKN37, because it lacks CAP, is heat shock resistant. Transformation of SKN37 with a plasmid expressing CAP restores heat shock sensitivity. The plasmid expressing profilin, by contrast, does not restore heat shock sensitivity to SKN37 (data not shown). These results indicate that profilin does not complement the $\mathrm{N}$-terminal functions of CAP.

\section{Organization of the Actin Cytoskeleton in CAP-Deficient Strains}

CAP-deficient strains have an abnormal distribution of actin that can be corrected by overexpression of CAP or profilin (Figure 3). cap- SKN32 cells transformed with the plasmid pCAP, which expresses the full-length CAP, exhibit the asymmetric actin distribution of a wild-type yeast cell (Adams and Pringle, 1984; Kilmartin and Adams, 1984). Actin is present in thin filaments or cables in the mother cell, arrayed longitudinally along the mother-bud axis, and in cortical patches in the bud (Figure 3A). This asymmetric actin distribution is not observed in SKN32 cells transformed with the control plasmid YEp13M4 (Figure 3B). Rather, in these cells actin is present in cortical patches in both mother cell and bud, and actin cables are absent. In a small percentage of cells, actin bars are observed. Transformation of SKN32 with pPFY, which expresses profilin, restores an asymmetric actin distribution (Figure 3C): cables, although fainter than in wild-type cells, are again visible in the mother cells, and cortical patches are primarily, but not exclusively, localized to the bud.

\section{Selection of Bud Sites in CAP-Deficient Strains}

In the yeast $\mathrm{S}$. cerevisiae, buds generally tend to emerge adjacent to previous bud sites. In haploid cells, the sites chosen are at one pole of the cell, whereas in diploid cells buds emerge from both poles (Sloat et al., 1981, and reierences cited therein). During budding, a ring of chitin is deposited at the neck of the bud; after cell division, the ring remains on the mother cell. Bud site selection can be readily assessed by staining these chitin-containing bud scars with the fluorescent dye Calcofluor. A ring of actin appears prior to chitin deposition and may play a role in bud site selection as well (Kilmartin and Adams, 1984).

CAP-deficient strains exhibit a random budding pattern that is corrected by overexpression of CAP and partially restored by overexpression of profilin (Figure 4 and Table 2). SKN32 cells transformed with pCAP primarily exhibit the wild-type haploid budding pattern: buds emerge adjacent to one another, as indicated by the clustered bud scars, and are at one pole of the cell (Figure 4A, left and center). Overexpression of CAP, however, leads to a 4-fold increase in bipolar budding, the typical pattern of $\mathbf{a} / \alpha$ cells (Figure 4A, right; Table 1). This increase in bipolar budding in haploid cells has also been observed on overexpression of the actin-binding protein ABP1 (Drubin et al., 1988).

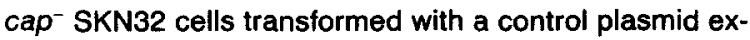
hibit a random budding pattern (Figure 4B). Expression of profilin partially restores the polar budding pattern (Figure $4 C$ ).

\section{Analysis of Profilin Gene Disruptions}

Disruption of the profilin gene has been reported to lead to morphological defects that are strikingly similar to those we find in cap ${ }^{-}$cells (Haarer et al., 1990). pty yeast are abnormally large and round, and the actin distribution resembles that seen in cap yeast except that pfy cells usually contain actin bars. Both pfy and cap cells show increased chitin delocalization as cells increase in size (data 
A
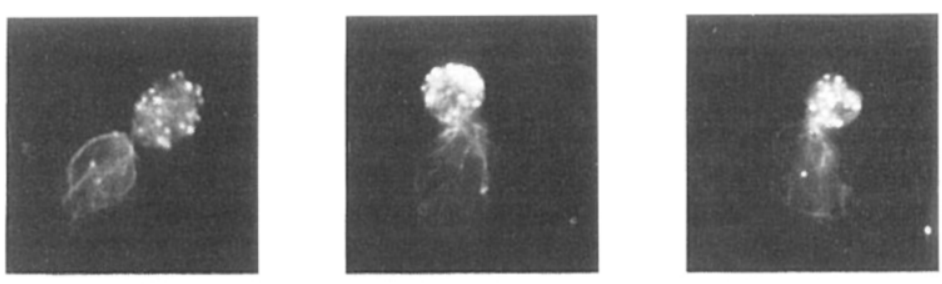

B

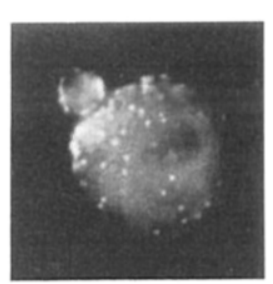

C

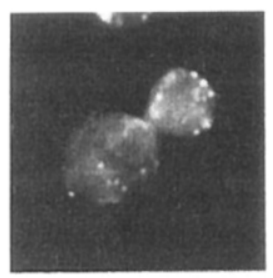

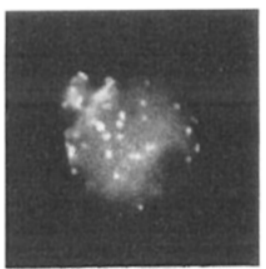

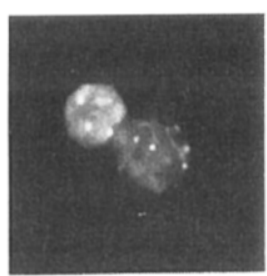

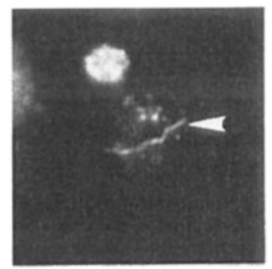

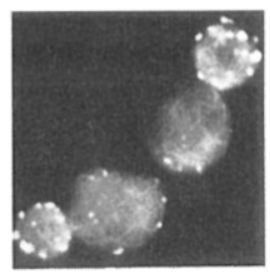

Figure 3. Effect of CAP or PFY on Actin Localization in cap Cells

SKN32 was transformed with PCAP (A) YEp13M4 (control) (B), or pPFY (C). Actin was visualized by indirect immunofluorescence. (The arrowhead denotes the location of an actin bar.) not shown; Haarer et al., 1990). pfy cells also display a random pattern of bud scars, grow more slowly than wild-type cells, and are temperature sensitive for growth. We have examined the growth characteristics of profilin disruptions in strains isogenic to our cap strains. One of the two copies of the profilin gene was disrupted in the diploid strain SP1/DC124 by integrating the $5.0 \mathrm{~kb}$ BamHIHindlll fragment from pSK-HBL (Haarer et al., 1990) or
A

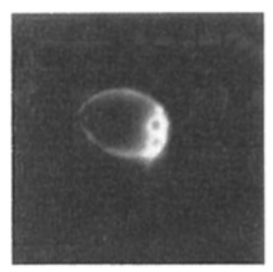

B

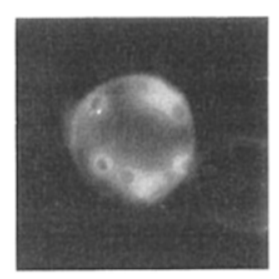

C

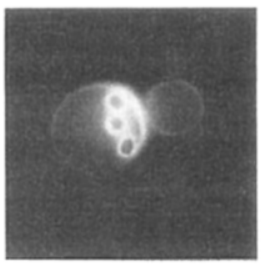

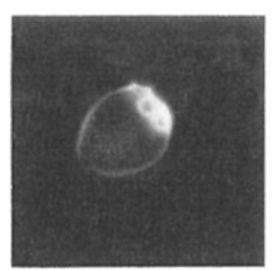
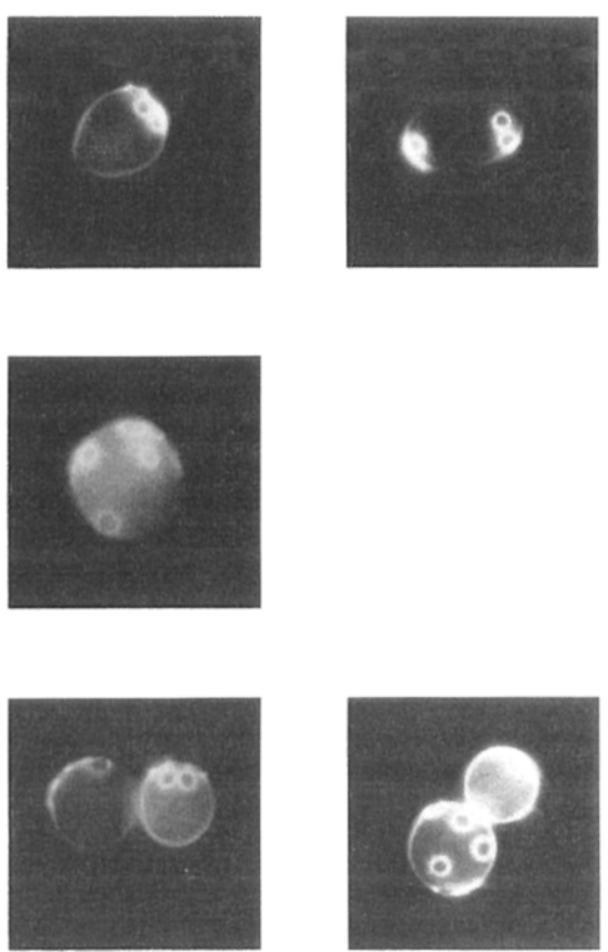

Figure 4. Effect of CAP or PFY on the Budding Pattern of cap Cells

SKN32 was transformed with PCAP (A), YEp13M4 (control) (B), or PPFY (C). Bud scars were visualized by staining with the fluorescent dye Calcofluor. 
Table 2. Effect of Multicopy CAP or PFY on Bud Site Selection in a cap- strain, SKN32.

\begin{tabular}{llcc}
\hline \multirow{2}{*}{ Strain } & \multicolumn{3}{l}{ Budding Pattern (\%) } \\
\cline { 2 - 4 } & Polar & Bipolar & Random \\
\hline SP1 (CAP') & 92 & 4.5 & 3.5 \\
SKN32 (cap $\left.{ }^{-}\right)$ & 20 & 0.5 & 79.5 \\
SKN32/pCAP & 79 & 16 & 5 \\
SKN32/pPFY & 63.5 & 1.5 & 35 \\
\hline
\end{tabular}

DCAP and pPFY are plasmids expressing full-length CAP and PFY genes, respectively. The budding pattern of various strains, grown in SC - Leu medium, was determined by staining cultures with Calcofluor and scoring at least 200 cells of each genotype with two or more bud scars.

the $1.2 \mathrm{~kb}$ BamHI-HindIII fragment from pU2-9. In both pSK-HBL and pU2-9 the profilin-coding sequences have been replaced with auxotrophic markers, LEU2 or URA3, respectively. Southern hybridization analysis confirmed that the fragments had integrated within one copy of the profilin gene. Diploid strains carrying these disruptions were subjected to tetrad analysis. Of the seven complete tetrads analyzed, the auxotrophic marker, URA3 or LEU2, cosegregated with the known pfy phenotypes: round and enlarged cells, temperature sensitivity, and slow growth on minimal medium. In addition, pfy haploid cell growth was slightly retarded on YPD in comparison with synthetic medium. This last growth defect appears to reflect sensitivity to increased concentrations of certain amino acids such as valine, and is similar to, but not nearly as severe as, the growth defect of cap strains. When $6.5 \mathrm{mM}$ valine is added to synthetic medium, growth of the cap strain SKN37 is totally inhibited, but the growth rate of the pfy strains is reduced only $30 \%-40 \%$ under these conditions (data not shown). The growth rate of wild-type strains is enhanced under these same conditions.
Table 3. Location and Identity of Profilin Amino Acid Substitutions

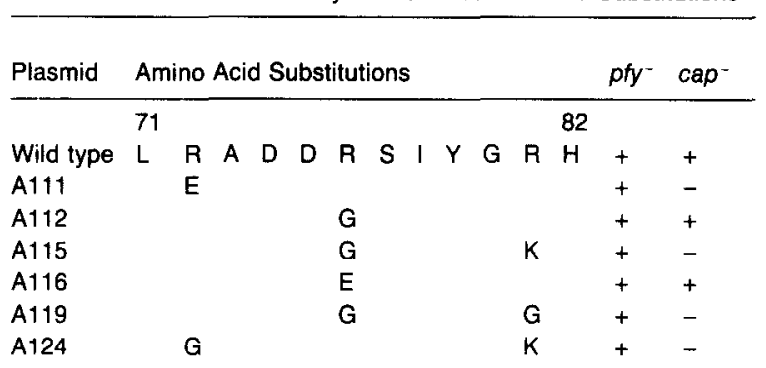

The sequence of the wild-type profilin is shown in the single-letter code. The plasmids contained wild-type or mutated PFY genes encoding the indicated amino acid substibutions. At right we indicate whether the designated plasmid was able to suppress $(+)$ or failed to suppress $(-)$ the phenotypic features of pfy- or cap- strains, PL1 and SKN32, respectively.

\section{Characterization of Profilin Point Mutations}

Yeast S. cerevisiae profilin most closely resembles the two profilins of Acanthamoeba (Figure 1B). These profilins contain several homology blocks, and the one between amino acid residues 71 and 82 of yeast profilin is particularly rich in positively charged amino acids. To distinguish those functions of profllin that are critical for suppression of a cap $^{-}$strain, we characterized a series of point mutations in arginine-72, arginine-76, and arginine-81 made in the S. cerevisiae profilin gene (Figure 5 and Table 3). Plasmids containing the point mutants of either or both arginine-72 and arginine-81 failed to suppress the phenotypes of the cap ${ }^{-}$strain (Figure 5). In addition, cap cells transformed with these profilin point mutants grow slower on synthetic medium than do cap cells transformed with the control vector YEp13M4 (data not shown). Mutations in arginine-76 alone were not sufficient to cause loss of activity in this assay.
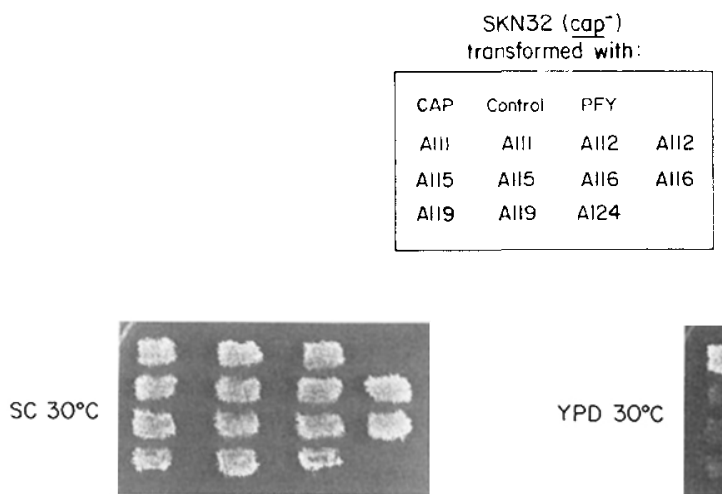

YPD $30^{\circ} \mathrm{C}$
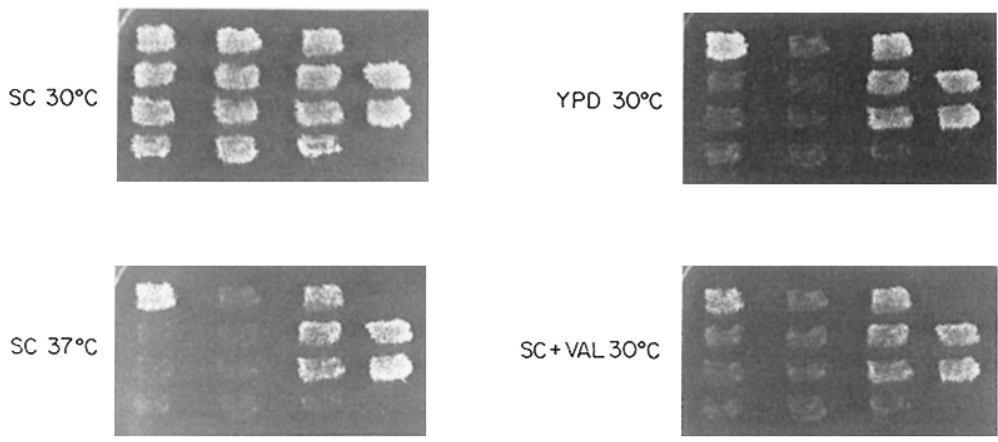

$S C+V A L 30^{\circ} \mathrm{C}$

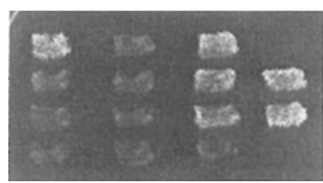

Figure 5. Effect of Profilin Mutants on cap Cells

SKN32 was transformed with the indicated plasmids (see Table 3 for mutant profilin sequences encoded by the A-series plasmids) and plated onto SC - Leu plates. Independent transformants were patched onto SC - LeU plates, incubated $3-4$ days at $30^{\circ} \mathrm{C}$, and then scored for growth on SC - Leu at $30^{\circ} \mathrm{C}$ (SC $30^{\circ} \mathrm{C}$ ); SC - Leu at $37^{\circ} \mathrm{C}\left(\mathrm{SC} 37^{\circ} \mathrm{C}\right)$; YPD at $30^{\circ} \mathrm{C}$; and $\mathrm{SC}-\mathrm{Leu}$ with $6.5 \mathrm{mM}$ valine at $30^{\circ} \mathrm{C}\left(\mathrm{SC}+\mathrm{VAL} 30^{\circ} \mathrm{C}\right)$. The control plasmid is YEp13M4. 


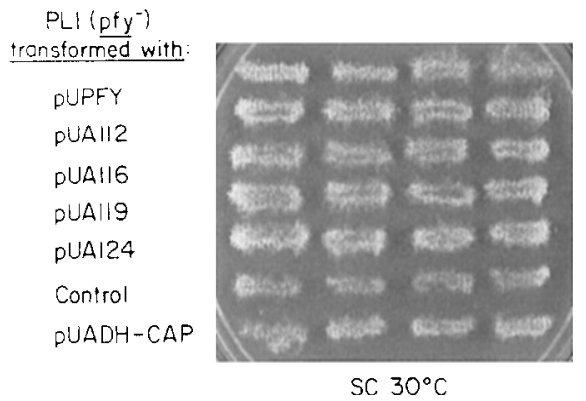

Sc $30^{\circ} \mathrm{C}$

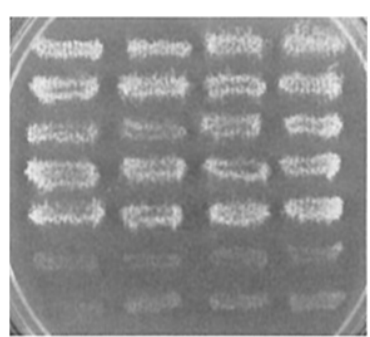

SC $37^{\circ} \mathrm{C}$
Figure 6. Suppression of a pfy Strain by Profilin Mutants

Strain PL1 was transformed with the indicated plasmids and plated onto SC - Ura plates. Independent transformants were patched onto SC - Ura plates, incubated $3-4$ days at $30^{\circ} \mathrm{C}$, and then replica plated to $\mathrm{SC}-\mathrm{Ura}$ at $30^{\circ} \mathrm{C}$ $\left(\mathrm{SC} 30^{\circ} \mathrm{C}\right)$ and SC.Ura at $37^{\circ} \mathrm{C}\left(\mathrm{SC} 37^{\circ} \mathrm{C}\right)$. The control plasmid is pUV2.
All the mutated profilins examined are comparable to wild-type profilin in their ability to suppress the slow growth and temperature sensitivity of the pfy strain (Figure 6). Moreover, all the point mutants bind to actin equally well when actin/profilin binding is assessed in vitro by Sepharose-bound DNAase I chromatography (B. H. and S. B., unpublished data). These results suggest that the ability of profilin to suppress the defects of a cap strain is dependent upon some additional function, perhaps one related to modulating a CAP-related signaling pathway.

\section{Expression of Acanthamoeba Profilins}

\section{In S. cerevlslae}

The protozoan Acanthamoeba contains two profilins, isoform I and isoform II, that bind actin with equal affinities (Kaiser et al., 1986). The affinity of isoform II for polyphosphoinositides, such as phosphatidylinositol 4,5-bisphosphate, is 10- to 50-fold higher than that of isolorm I (Machesky et al., 1990), and therefore we compared the two profilin isoforms for their abilities to suppress defects in cap- strains. The Acanthamoeba profilins were expressed in yeast and tested by a replica-plating method for their ability to restore growth on rich medium to a cap strain. As shown in Figure 7, isoform II, but not isoform I, restores growth on rich medium to SKN37, although not as well as the wild-type yeast profilin. On prolonged incubation, strains transformed with isoform I grow weakly on rich medium. On the other hand, both isoform I and isoform II suppress the growth defects of a pfy strain (Figure 8). At higher temperatures, isoform I suppresses somewhat better than isoform II. In addition, isoform I appears to be present at higher levels than isoform II (B. H. and S. B., unpublished data). These results are consistent with the idea that suppression of a cap ${ }^{-}$strain is dependent upon some additional function of profilin besides actin binding, and further suggest that this additional function may require an interaction with polyphosphoinositides.

\section{Discussion}

We have found that multicopy plasmids expressing profilin can suppress morphological and nutritional defects in cells lacking CAP. Only the functions due to loss of the C-terminus of CAP are corrected. The function of the $\mathrm{N}$-terminus of CAP, namely, cellular responsiveness to the activated mutant RAS2val19, is not restored by overexpression of profilin. The phenotypes of cells without profilin closely resemble the phenotypes of cells lacking the C-terminus of CAP (see Table 4): such cells are round and heterogeneously large, are often multinucleated, bud randomly from the cell surface, have aberrant actin distribution, are temperature sensitive, and show growth defects under certain nutritional conditions. Moreover, we have identified mutants of profilin that aggravate the CAP-deficient phenotype even in the presence of wild-type profilin. Altogether, these results indicate that CAP and profilin shape similar cellular events, and suggest that CAP and profilin operate on the same pathway.

CAP and profilin deficiencies both lead to randomized bud site selection. Several other yeast mutants with defects in budding have been previously described. These "bud" mutants can be classified into two groups: those

\begin{tabular}{lll}
\hline Table 4. Comparison of cap- and pty- & \\
\hline & Seast Strains & \\
\hline Phenotype & $c a p^{-}$ & $p^{-}$ \\
\hline Growth & & \\
Synthetic medium & Slow & Slow \\
Rich medium & None & Reduced \\
Temperature sensitivity & Yes & Yes \\
Valine toxicity & Strong & Weak \\
Cell morphology & Enlarged, round, multinucleated & Enlarged, round, multinucleated \\
Actin distribution & No cables & No cables \\
& Cortical patches & Cortical patches \\
Budding pattern & Actin bars: rare & Actin bars: common \\
Chitin distribution & Random & Random \\
\hline
\end{tabular}


SKN37 ( cop $\left.^{-}\right)$

transformed with

S. cerevisıa PFY

Control

Aconthamoebo PFY PI

Aconthomoebo PFY PI

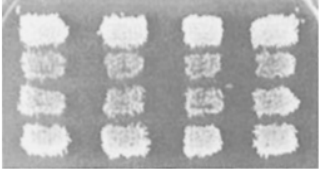

$\mathrm{SC} 30^{\circ} \mathrm{C}$

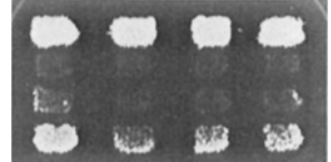

YPD $30^{\circ} \mathrm{C}$
Figure 7. Effect of Acanthamoeba Profilins on cap Cells

SKN37 was transformed with the indicated plasmids and plated onto SC - Ura plates. Independent transformants were patched onto $\mathrm{SC}$ - Ura plates, incubated at $30^{\circ} \mathrm{C}$, and then replica plated to SC - Ura (SC) and YPD at $30^{\circ} \mathrm{C}$ (bud1-bud5) that affect merely bud site selection (Chant and Herskowitz, 1991; Chant et al., 1991), and those (cdc24, cdc42, cdc43, and cap2) that all exhibit various aspects of the morphological defects seen in CAP- and profilin-deficient cells (Sloat et al., 1981; Adams et al., 1990; Amatruda et al., 1990). To date, none of the mutants of this latter group have been reported to display the sensitivities to growth conditions that typify CAP-deficient cells, nor have any of these genes been isolated as suppressors of CAP deficiencies in our screens of multicopy yeast genomic DNA. It will therefore be of interest to test these mutants for additional growth defects. Even though profilin mutants have only mild nutritional defects, the observation that overexpression of profilin can reverse the nutritional defects of CAP strains indicates that, like CAP, profilin can participate in two pathways: one affecting cytoskeletal function, the other affecting growth and viability under nutritional stress.
We have identified point mutants of profilin that seem to dissociate these two pathways. Profilins with amino acid substitutions at either arginine-72 or arginine-81 are not apparently altered in their ability to bind actin (B. H. and S. B., unpublished data), and their expression suppresses the loss of wild-type profilin, as shown here. However, expression of these mutant profilins does not suppress the loss of CAP. Thus, the ability of profilins to suppress loss of CAP may not be solely dependent on their cytoskeletal interactions, but may require an additional function, perhaps the ability to bind and modulate the hydrolysis of polyphosphoinositides.

Studies of the Acanthamoeba profilins support the idea that polyphosphoinositide binding is necessary for suppression of the CAP-deficient phenotypes. The two Acanthamoeba profilin isoforms, which differ at only a handful of residues, bind actin equally well (Kaiser et al., 1986), and we have shown that each is capable of suppressing

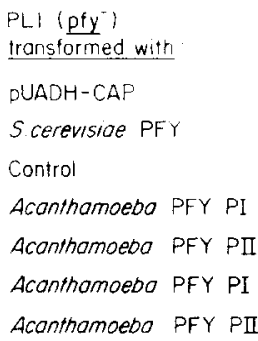

Figure 8. Suppression of pfy Strain by Acanthamoeba Profilins

Strain PL1 was transformed with the indicated plasmids and plated onto SC - Ura plates. Independent transformants were patched onto $\mathrm{SC}$ - Ura plates, incubated at $30^{\circ} \mathrm{C}$, and then replica plated to SC - Ura (SC) and YPD and incubated at the specified temperatures. RT room temperature.
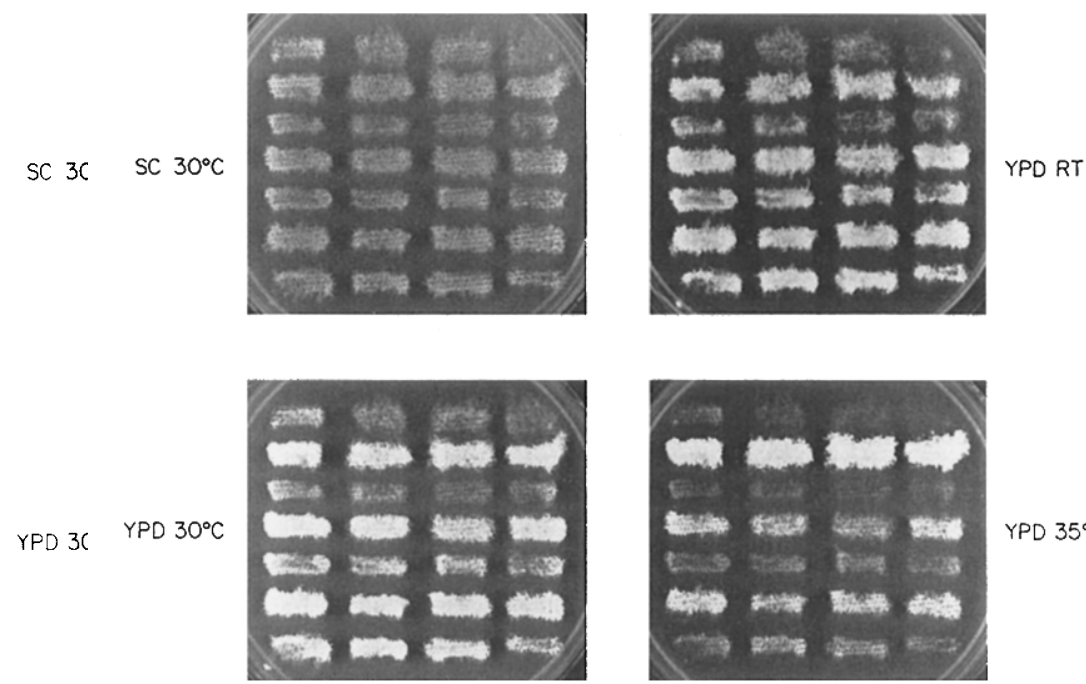
the loss of wild-type profilin in S. cerevisiae. However, isoform II binds polyphosphoinositides with 10- to 50-fold greater affinity than does isoform I (Machesky et al., 1990) and is superior to isoform $I$ in suppressing the growth defects of CAP-deficient cells.

These observations lead us to suggest that loss of CAP alters the formation of second messengers resulting from phosphoinositide metabolism, and that these second messengers are important in regulating cellular morphology and cellular adaptation to a variety of nutritional environments. Our data are consistent with a large number of specific models. Further biochemical and genetic analysis is required to resolve these models. Alterations in the metabolism of phosphoinositides might be apparent in mutants of either CAP or profilin, although significant changes in subcompartments of metabolites might not be measurable. If CAP affects phosphoinositide metabolism, then the expression of genes encoding phospholipases or phospholipid kinases might alter the CAP-deficient phenotype. It would be useful to characterize the precise biochemical defect of mutant profilins bearing substitutions at arginine-72 and arginine-81, or to demonstrate the existence of mutant profilins capable of suppressing loss of CAP function that cannot suppress loss of profilin function. Such studies are in progress.

Our experiments bring together multiple regulatory pathways in an unexpected fashion. CAP may somehow coordinate nutritional signaling with cytoskeletal rearrangements. We have no evidence that RAS controls CAP, but in yeast both RAS and CAP appear to interact with at least one common target, adenylyl cyclase. Although the relationship between RAS and adenylyl cyclase has not been maintained in mammalian cells, it is possible that a relationship between RAS, CAP, and profilin has been maintained in evolution. Identification of a mammalian CAP homolog or the heterologous expression of the S. cerevisiae CAP gene in mammalian cells may prove useful in establishing such a relationship.

\section{Experimental Procedures}

\section{Strains, Media, and Genetic Manipulations}

Yeast strains are described in Table 1. Yeast were grown on synthetic medium (SC; $0.67 \%$ yeast nitrogen base with ammonium sulfate, $3 \%$ glucose, and appropriate auxotrophic supplements) or on YPD (1\% yeast extract, $2 \%$ Bacto-Peptone, $3 \%$ glucose) agar plates. Strains lacking $C A P$ were grown on plates at $30^{\circ} \mathrm{C}$, or in liquid cultures at room temperature; strains lacking profilin were grown at room temperature unless otherwise specified. Standard methods for yeast transformation, gene disruption, and tetrad dissection were employed (Rose et al., 1990). Replica plating methods were used to assay for sensitivity to growth on rich medium, temperature, heat shock, nitrogen starvation, and valine (Field et al., 1990; Gerst et al., 1991).

\section{DNA Manipulations}

Restriction endonucleases, ligases, and polymerases were purchased from New England Biolabs or Perkin-Elmer Cetus. Standard techniques and conditions were used (Maniatis et al., 1982). DNA was sequenced by the chain termination method using Sequenase version 2.0 according to the manufacturer's directions (United States Biochem. ical Corporation).

\section{Plasmids}

pPFY is a genomic profilin clone isolated from a library in the yeast vector YEp13M4 (J. Nikawa, unpublished data) by complementation of the sensitivity of SKN37 to growth on rich medium. pUPFY is a genomic profilin clone isolated from a library in the yeast vector PUV1 (J. Nikawa, unpublished data) by complementation of the YPD sensitivity of SKN37. Restriction map and Southern hybridization analysis indicated that pUPFY encoded profilin. PCAP is identical to pYEPCAP, the genomic CAP clone described previously (Field et al., 1990). pUADH-CAP expresses the CAP gene under control of the ADH1 promoter (Gerst et al., 1991).

\section{Plasmid Constructions}

The A-series profilin mutants were constructed by site-directed mutagenesis of profilin using the Bio-Rad (Richmond, CA) in vitro mutagenesis kit following the manufacturer's instructions. Single-stranded template DNA was produced from $\mathrm{M} 13 \mathrm{mp} 19$ containing the entire profilin gene on a $1.8 \mathrm{~kb}$ BamHI-HindlII fragment; $M+3$ was propagated in $J M 103$. The following degenerate 49 base primer, corresponding to nucleotides 199 to 247 of the profilin-coding region, was used to introduce changes at basic residues: (A/G)AGTTCATGTTGTTG(AVG)(A G)AGCTGACGAT(A)(AVG)AAGTATCTACGGT(AG)(AVG)A(C/G)ATG. Altered profilin genes were identified by sequencing. In some cases, products of mutagenesis were screened by plaque hybridization using the following primer (corresponding to nucleotides 214 to 228 of the profilin coding region): AGAGCTGACGATAGA. Mutated profilin genes were isolated from the $M 13$ vector as $1.8 \mathrm{~kb}$ BamHI-HindIII fragments and inserted into the BamHI-HindIII sites of YEp102 and YEp13M4. (YEp102 consists of the $2 \mu$-containing $2.2 \mathrm{~kb}$ EcoRl fragment from YEp24 [Botstein et al., 1979] inserted into the EcoRI site of YIp5 [Botstein et al., 1979].)

The Acanthamoeba profilin fusion genes were generated as described below. Full-length cDNAs encoding Acanthamoeba profilin isoform I (PI) and isoform II (PII) were cloned as $\sim 700$ bp EcoRI fragments in pBluescript KS+ (Stratagene, La Jolla, CA) (Pollard and Rimm, 1991). The coding regions for these genes were placed next to the yeast profilin promoter as follows: First, a 500 bp Sall-Pvul fragment containing $5^{\prime}$ noncoding sequences from the yeast profilin gene was blunt-ended with mung bean nuclease (to generate a fragment spanning positions -508 to -11 ) and inserted into the blunt-ended (with Klenow) Xbal sites of the PII-containing plasmid (Xbal digestion removes all but 12 bases of the $5^{\prime}$ noncoding region of PII). The Pvul-Xbal junction of the resulting plasmid (PFY-PII) was confirmed by sequencing. Second, the corresponding yeast promoter-profilin I plasmid (PFY-PI) was generated by replacing the PII coding region, from a Sall site at codon 7 through the end of the CDNA, with the corresponding region from the PI clone. PI and PII cDNA sequences are identical from the initiator ATG through this Sall site. Third, the inserts of plasmids PFY-PI and PFY-PII were moved as Eagl-Xhol fragments to the yeast vector YEp102 to generate plasmids YEp(PFY-PI) and YEp(PFY-PII).

The profilin gene disruption plasmid, pU2-9, was constructed by deleting the coding region of profilin, using the polymerase chain reaction (PCR), and replacing it with the URA3 auxotrophic marker. The template for the PCR was pP1-7B2, which contains the profilin gene and $5^{\prime}$ and $3^{\prime}$ flanking sequences on a $1.8 \mathrm{~kb}$ BamHI-Hindlll fragment in pUC119. The PCR utilized an oligonucleotide primer complementary to the upstream flanking sequence of profilin, 5'-CTAGGATATCCCAAGACATAATTTGC-3', and one complementary to the downstream flanking region of profilin, 5'-TACTGATATCGTGTTCAATACTAAT-3; both primers contain an EcoRV restriction endonuclease site. The $3^{\prime}$ ends of both primers were oriented outward from the profilin coding sequence so as to amplify a $4.5 \mathrm{~kb}$ region containing only the $5^{\prime}$ and $3^{\prime}$ flanking sequences of profilin (but not the profilin coding sequence) and the vector backbone. The PCR product was purified by gel electrophoresis, digested with EcoRV, and ligated to generate plasmid pP2. In plasmid pP2, the profilin coding sequence between nucleotides +9 and +340 was deleted and an EcoRV site inserted at the site of the deletion as a result of the PCR and subsequent steps. The Klenowtreated $1.1 \mathrm{~kb}$ HindIII URA3 gene fragment was then inserted into the EcoRV site of pP2 to generate pU2-9.

\section{Fluorescence Mlcroscopy Methods}

Actin was visualized with affinity-purified rabbit anti-actin primary antibody (Haarer et al., 1990) and FITC-conjugated goat anti-rabbit IgG secondary antibody (Sigma), as described (Pringle et al., 1989). Chitin was stained with the fluorescent dye Calcofluor (Pringle et al., 1989). 


\section{Acknowledgments}

We thank Dave Turner for insightful discussions, and Patricia Bird for assistance in preparing the manuscript. This work was supported by grants from the National Institutes of Health (T. D. P., B. H., M. W.), the American Cancer Society (S. B., M. W.), and the National Cancer Institute (M. W. A. V.). M. W. is an American Cancer Society Research Professor.

The costs of publication of this article were defrayed in part by the payment of page charges. This article must therefore be hereby marked "advertisement" in accordance with 18 USC Section 1734 solely to indicate this fact.

Received April 18, 1991; revised June 6, 1991.

\section{References}

Adams, A., and Pringle, J. (1984). Relationship of actin and tubulin distribution in wild-type and morphogenetic mutant Saccharomyces cerevisiae. J. Cell Biol. 98, 934-945.

Adams, A., Johnson, D., Longnecker, R., Sloat, B., and Pringle, J. (1990). CDC42 and CDC43, two additional genes involved in budding and the establishment of cell polarity in the yeast Saccharomyces cerevisiae. J. Cell Biol. 111, 131-142.

Amatruda, J., Cannon, J., Tatchell, K., Hug, C., and Cooper, J. (1990). Disruption of the actin cytoskeleton in yeast capping protein mutants. Nature 344, 352-354.

Barbacid, M. (1987). ras Genes. Annu. Rev. Biochem. 56, 779-827. Berridge, M., Hesiop, J., Ivvine, R., and Brown, K. (1984). Inositol trisphosphate formation and calcium mobilization in Swiss 3T3 cells in response to platelet-derived growth factor. Biochem. J. 222, 195-201. Botstein, D., Falco, S., Stewart, S., Brennan, M., Scherer, S., Stinchcomb, D., Struhl, K., and Davis, R. (1979). Sterile host yeasts (SHY) a eukaryotic system of biological containment for recombinant DNA experiments. Gene 8, 17-24.

Chant, J., and Herskowitz, I. (1991). Genetic control of bud site selection in yeast by a set of gene products that constitute a morphogenetic pathway. Cell 65, 1203-1212.

Chant, J., Corrado, K., Pringle, J. R., and Herskowitz, I. (1991). Yeast BUD5, encoding a putative GDP-GTP exchange factor, is necessary for bud site selection and interacts with bud formation gene BEM1. Cell 65, 1213-1224.

Drubin, D., Miller, K., and Botstein, D. (1988). Yeast actin-binding proteins: evidence for a role in morphogenesis. J. Cell Biol. 107, 25512561.

Fedor-Chaiken, M., Deschenes, R. J., and Broach, J. R. (1990). SRV2, a gene required for $R A S$ activation of adenylate cyclase in yeast. Cell $61,329-340$

Field, J., Vojtek, A., Ballester, A., Bolger, G., Colicelli, J., Ferguson, K., Gerst, J., Kataoka, T., Michaeli, T., Powers, S., Riggs, M., Rodgers, L., Wieland, I., Wheland, B., and Wigler, M. (1990). Cloning and characterization of CAP, the $\mathrm{S}$. cerevisiae gene encoding the $70 \mathrm{kd}$ adenylyl cyclase-associated protein. Cell 61, 319-327.

Gerst, J., Ferguson, K., Vojtek, A., Wigler, M., and Field, J. (1991). CAP: a bifunctional component of the $S$. cerevisiae adenylyl cyclase complex. Mol. Cell. Biol. 11, 1248-1257.

Goldschmidt-Clermont, P., Machesky, L., Baldassare, J., and Pollard, $T$. (1990). The actin-binding protein profilin binds to $\mathrm{PIP}_{2}$ and inhibits its hydrolysis by phospholipase C. Science 247, 1575-1578

Goldschmidt-Clermont, P., Kim, J., Machesky, L., Rhee, S., and Pollard, T. (1991). Regulation of phospholipase C- $\gamma 1$ by profilin and tyrosine phosphorylation. Science 251, 1231-1233.

Haarer, B., Lillie, S., Adams, A., Magdolen, V., Bandlow, W., and Brown, S. (1990). Purification of profilin from Saccharomyces cerevisiae and analysis of profilin-deficient cells. J. Cell Biol. 110, 105-114. Janmey, $P$., and Stossel, T. (1987). Modulation of gelsolin function by phosphatidylinositol 4,5-bisphosphate. Nature 325, 362-364.

Kaiser, D., Sato, M., Ebert, R., and Pollard, T. (1986). Purification and characterization of two isoforms of Acanthamoeba profilin. J. Cell Biol. 102, 221-226.
Kilmartin, J., and Adams, A. (1984). Structural rearrangements of tubulin and actin during the cell cycle of the yeast Saccharomyces. J. Cell Biol. 98, 922-933.

Lassing, I., and Lindberg, U. (1985). Specific interaction between phosphatidylinositol 4,5-bisphosphate and profilactin. Nature 314, 472474.

Lassing, I., and Lindberg, U. (1988). Specificity of the interaction between phosphatidylinositol 4,5-bisphosphate and the profilin:actin complex. J. Cell. Biochem. 37, 255-267.

Machesky, L., Goldschmidt-Clermont, P., and Pollard, T. (1990). The affinities of human platelet and Acanthamoobe profilin isoforms for polyphosphoinositides account for their relative abilities to inhibit phospholipase C. Cell Reg. 1, 937-950.

Magdolen, V., Oechsner, U., Müller, G., and Bandlow, W. (1988). The intron-containing gene for yeast profilin (PFY) encodes a vital function. Mol. Cell. Biol. 8, 5108-5115.

Maniatis, T., Fritsch, E. F., and Sambrook, J. (1982). Molecular Cloning: A Laboratory Manual (Cold Spring Harbor, New York: Cold Spring Harbor Laboratory).

Margolis, B., Rhee, S. G., Felder, S., Mervic, M., Lyall, R., Levitzki, A., Ullrich, A, Zilberstein, A, and Schlessinger, J. (1989). EGF induces tyrosine phosphorylation of phospholipase C-Il: a potential mechanism for EGF receptor signaling. Cell 57, 1101-1107.

Meisenhelder, J., Suh, P.-G., Rhee, S. G., and Hunter, T. (1989). Phospholipase $C-\gamma$ is a substrate for the PDGF and EGF receptor proteintyrosine kinses in vivo and in vitro. Cell 57, 1109-1122.

Pollard, T., and Cooper, J. (1986). Actin and actin-binding proteins. A critical evaluation of mechanisms and functions. Annu. Rev. Biochem. 55, 987-1035.

Pollard, T. D., and Rimm, D. L. (1991). Analysis of CDNA clones for Acanthamoeba profilin-I and profilin-\|l shows end to end homology with vertebrate profilins and a family of profilin genes. Cell Motil. Cytoskel., in press.

Pringle, J., Preston, R., Adams, A., Stearns, T., Drubin, D., Haarer, B., and Jones, E. (1989). Fluorescence microscopy methods for yeast. Meth. Cell Biol. 31, 357-435.

Rose, M., Winston, F., and Hieter, P. (1990). Methods in Yeast Genetics: A Laboratory Course Manual (Cold Spring Harbor, New York: Cold Spring Harbor Laboratory).

Sloat, B., Adams, A., and Pringle, J. (1981). Roles of the CDC24 gene product in cellular morphogenesis during the Saccharomyces cerevisiae cell cycle. J. Cell Biol. 89, 395-405.

Stossel, T., Chaponnier, C., Ezzell, R., Hartwig, J., Janmey, P., Kwiathkowski, D., Lind, S., Smith, D., Southwick, F., Yin, H., and Zaner, K. (1985). Nonmuscle actin-binding proteins. Annu. Rev. Cell Biol. 1, 353-402.

Toda, T., Uno, I., Ishikawa, T., Powers, S., Kataoka, T., Broek, D., Cameron, S., Broach, J., Matsumoto, K., and Wigler, M. (1985). In yeast, RAS proteins are controlling elements of adenylate cyclase. Cell 40, 27-36.

Wahl, M., and Carpenter, G. (1988). Regulation of epidermal growth factor-stimulated formation of inositol phosphates in A-431 cells by calcium and protein kinase C. J. Biol. Chem. 263, 7581-7590.

Wahl, M., Daniel, T., and Carpenter, G. (1988). Antiphosphotyrosine recovery of phospholipase C activity after EGF treatment of A-431 cells. Science 241, 968-969.

Wahl, M., Nishibe, S., Suh, P.-G., Rhee, S., and Carpenter, G. (1989) Epidermal growth factor stimulates tyrosine phosphorylation of phospholipase C-II independently of receptor internalization and extracellular calcium. Proc. Natl. Acad. Sci. USA 86, 1568-1572. 